\title{
Hispanismes
}

Revue de la Société des Hispanistes Français

\section{La communauté hispanophone en Suisse romande et ses pratiques linguistiques}

Étude en cours avec le Corpus oral de la lengua española en la Suiza francófona (COLESfran)

La comunidad hispanohablante en Suiza y sus prácticas lingüísticas. Estudio en curso con el Corpus oral de la lengua española en la Suiza francófona

The Spanish-speaking community in French-speaking Switzerland and its linguistic practices. Ongoing study with the Corpus oral de la lengua Española en la Suiza francófona (COLESfran)

\section{Mónica Castillo Lluch}

\section{CpenEdition}

\section{Journals}

\section{Édition électronique}

URL : https://journals.openedition.org/hispanismes/885

DOI : 10.4000/hispanismes.885

ISSN : 2270-0765

Éditeur

Société des Hispanistes Français

\section{Référence électronique}

Mónica Castillo Lluch, «La communauté hispanophone en Suisse romande et ses pratiques linguistiques », Hispanismes [En ligne], 16 | 2020, mis en ligne le 01 décembre 2020, consulté le 31 août 2021. URL : http://journals.openedition.org/hispanismes/885; DOI : https://doi.org/10.4000/ hispanismes.885

Ce document a été généré automatiquement le 31 août 2021.

Les contenus de cette revue sont mis à disposition selon les termes de la Licence Creative Commons Attribution - Pas d'Utilisation Commerciale - Pas de Modification 4.0 International. 


\section{La communauté hispanophone en Suisse romande et ses pratiques linguistiques}

Étude en cours avec le Corpus oral de la lengua española en la Suiza francófona (COLESfran)

La comunidad hispanohablante en Suiza y sus prácticas lingüísticas. Estudio en curso con el Corpus oral de la lengua española en la Suiza francófona

The Spanish-speaking community in French-speaking Switzerland and its

linguistic practices. Ongoing study with the Corpus oral de la lengua Española en la Suiza francófona (COLESfran)

Mónica Castillo Lluch

\section{Introduction}

1 Cet article présente un projet d'interviews sociolinguistiques que nous menons depuis 2013 à l'Université de Lausanne en vue d'explorer différents aspects du bilinguisme de la communauté espagnole et hispano-américaine en Suisse romande ${ }^{1}$. L'ensemble de ces interviews constitue le Corpus oral de la lengua española en la Suiza francófona (COLESfran) et se prête à une analyse linguistique selon une double approche: sociologique et structurelle. Du point de vue de la sociologie des langues dans le contexte de la migration, le corpus permet de connaître le processus d'intégration linguistique de cette communauté en Suisse et ses attitudes envers les langues d'origine et d'accueil ; d'un point de vue structurel, il révèle l'influence que le français exerce sur l'espagnol de ces locuteurs bilingues.

Dans les pages suivantes, tout d'abord sera dressé un bref profil démographique et historique de la communauté espagnole et hispano-américaine résidant en Suisse (romande) (\$1), seront ensuite exposés les objectifs, la méthodologie du CoLESfran, son état actuel et les défis auxquels il est confronté (\$2), sera enfin présenté l'intérêt du 
corpus en tant qu'outil pédagogique, ainsi que certains de ses résultats et perspectives (\$3), avant quelques brèves conclusions.

\section{Démographie et histoire de la communauté hispanophone en Suisse romande}

3 Dans le petit pays du continent européen qu'est la Suisse (environ $41.000 \mathrm{~km} 2$ et 8.600.000 habitants en 2019), le nombre de résidents espagnols et hispano-américains est remarquable et devient proportionnellement exceptionnel par rapport aux pays voisins. Si l'on considère notamment le nombre de résidents espagnols, il s'agit en fait, par rapport à la population dans son ensemble, du plus élevé de tous les pays choisis par les Espagnols comme destination migratoire. Alors qu'en 2019, en France, en Allemagne ou au Royaume-Uni, les Espagnols étaient respectivement 273 290, 167151 et 152291 - ce qui correspond à $0,4 \%, 0,2 \%, 0,23 \%$ de la population -, en Suisse, ils étaient 124 414, soit $1,4 \%^{2}$. En Suisse romande, ce pourcentage augmente et dans des villes comme Lausanne et Genève, il atteint $3,5 \%$ et $3,9 \%$ des habitants respectivement ${ }^{3}$, ce qui signifie qu'un Lausannois sur 28 et un Genevois sur 25 est espagnol (voire un Lausannois sur 20 et un Genevois sur 18 si l'on compte les personnes binationales). Dans ces deux villes, en $2018,7,5 \%$ et $9,6 \%^{4}$ ont déclaré que l'espagnol était l'une de leurs langues principales, soit une personne sur 13 à Lausanne et une sur 10 à Genève. Avec ces taux d'hispanophones (espagnols notamment), le nord-ouest du lac Léman se distingue dans la géographie suisse, européenne et mondiale.

4 La Suisse a commencé à attirer les Espagnols dans le contexte des Trente Glorieuses, plus précisément à partir de 1961, lorsque l'Espagne a conclu avec ce pays un accord bilatéral d'immigration ${ }^{5}$. La main-d'œuvre espagnole est dès lors devenue une alternative à la main-d'œuvre italienne, qui avait pris de l'importance dans les années 50, mais depuis 1960 était devenue moins attrayante en raison des exigences du gouvernement italien, qui voulait de meilleures conditions pour ses travailleurs dans la Confédération suisse. En 1970, le nombre d'Espagnols est passé à 121239 entre résidents permanents et temporaires. Le bien-être économique et social du pays explique pourquoi ce chiffre n'a baissé qu'en 1990, coïncidant avec la période de plus grand développement économique de l'Espagne. En 2010, le nombre d'Espagnols résidents permanents représentait la moitié de celui des années 70 (64 126), mais, à partir de cette date et en raison de la nouvelle crise économique mondiale, le nombre de migrations a de nouveau augmenté, pour atteindre 84.362 résidents permanents mono-nationaux en 2019 (selon les données de l'OFS, lesquelles augmentent de $47 \%$ pour l'INE (124 414), qui inclut les résidents binationaux).

5 La migration vers la Suisse des Hispano-américains a commencé dans les années 1970 avec l'exil politique des Chiliens et des Argentins et s'est progressivement accrue au cours des décennies suivantes pour atteindre 32.836 résidents permanents en 2019, selon l'OFS ${ }^{6}$. À ce nombre d'Hispano-américains en Suisse, il faut ajouter ceux qui dans les statistiques sont considérés comme Espagnols, car ils ont obtenu un passeport en Espagne à la suite d'une période migratoire dans ce pays ${ }^{7}$. Par conséquent, il convient de noter que l'écart entre le nombre d'Espagnols et d'Américains d'origine hispanique est désormais bien moins important que par le passé. La figure 1 montre la dynamique migratoire qui vient d'être décrite ${ }^{8}$. 
Fig. 1 : Ressortissants des pays hispanophones vivant en Suisse

Compilé à partir des données de l'OFS

Comme déjà mentionné, la Suisse romande (cantons de Genève, Vaud, Neuchâtel, Jura et une partie du Valais, Fribourg et Berne), attire un nombre très important d'hispanophones. Un regard sur les chiffres de la population suisse qui désigne l'espagnol comme étant l'une de ses langues principales en 2018 montre que cette population se répartit entre la Suisse alémanique, la Suisse romande et la Suisse italienne dans des proportions de $53 \%, 43 \%$ et $4 \%$ respectivement (voir figure 2). Étant donné que la région francophone représente $25 \%$ du pays en termes démographiques et économiques (le PIB de la région couvre $1 / 4$ du PIB national en 2019), cette concentration de $43 \%$ de la population hispanophone ici est surdimensionnée par rapport aux autres régions et pourrait raisonnablement s'expliquer par la préférence de nombreux immigrants hispanophones pour une zone de langue romane, où leur intégration linguistique, et par conséquent sociale, est envisagée comme plus facile. Cette répartition du nombre total d'hispanophones en Suisse est restée stable depuis 1970 , avec de légères variations.

Fig. 2 : Répartition de la population totale de 15 ans et plus désignant l'espagnol comme étant l'une de leurs langues principales par région linguistique en 2018



Élaboration propre à partir de l'Atlas statistique de la Suisse : Les 4 régions linguistiques de la Suisse par commune, en 2016 de l'OFS ${ }^{9}$ et de données fournies par l'OFS.

Depuis 60 ans, on observe donc plusieurs dynamiques au sein de la communauté hispanophone de Suisse (romande), dont trois sont particulièrement remarquables : 1) à la première génération de migrants économiques espagnols arrivés au siècle dernier, alloglotte en Suisse, se sont ajoutées les deuxième et troisième générations, qui maîtrisent mieux la langue suisse correspondant à leur canton de résidence que l'espagnol ; 2) le nombre d'hispanophones a considérablement augmenté au cours des trois dernières décennies, entraînant la coexistence de divers dialectes de la langue espagnole; et 3) depuis 2010, on assiste à un nouveau mouvement migratoire avec des professionnels généralement très qualifiés et polyglottes maîtrisant bien l'anglais. 
8 Dans le but d'explorer l'utilisation des langues par la communauté hispanophone de Suisse romande, nous avons entrepris depuis 2013 à l'Université de Lausanne (UNIL) le projet du Corpus oral de la lengua española en la Suiza francófona (COLESfran), qui est décrit dans la section suivante.

\section{Objectifs, méthodologie, contenus et défis du COLESfran}

9 Le Corpus oral de la lengua española en la Suiza francófona (COLESfran) recueille des témoignages oraux d'hispanophones vivant en Suisse romande afin de pouvoir les analyser sous la double perspective de la sociologie des langues dans le contexte de la migration et de la structure linguistique, en tenant compte des particularités de la langue espagnole qui sont le produit du contact avec le français.

10 Du point de vue de la sociologie des langues dans le contexte de la migration, ce corpus vise à découvrir, tout d'abord, comment s'est déroulé le processus d'intégration sociolinguistique des hispanophones de première génération et quel a été l'impact sur ce processus de variables telles qu'un éventuel bilinguisme antérieur, l'activité professionnelle, le couple (hispanophone ou francophone), la famille, les amis, les relations avec l'Espagne et l'accès à des cours de français. Un deuxième objectif consiste à analyser les attitudes linguistiques de ces hispanophones envers leurs enfants et petits-enfants et l'évolution possible dans le temps de leur représentation de l'espagnol et du français. En ce qui concerne les deuxième et troisième générations, le COLESfran sert à explorer comment elles développent des compétences en espagnol, leur utilisation des cours de langue et de culture d'origine ${ }^{10}$ et leurs représentations sur leur langue d'héritage. En outre, en raison des relations que peuvent établir en Suisse des hispanophones originaires de régions très éloignées et donc dialectalement différentes, il est important de connaître les représentations que ces locuteurs ont de leur variété et celles des autres locuteurs, ainsi que les attitudes qu'ils manifestent dans les situations d'interaction avec des hispanophones d'autres régions, en particulier s'ils déclarent converger ou non avec les dialectes de leurs interlocuteurs.

11 D'un point de vue sociolinguistique, le COLESfran prétend servir comme base de données pour analyser l'espagnol de différentes générations d'hispanophones résidant en Suisse romande et notamment les phénomènes de contact avec le français qui le façonnent ${ }^{11}$. L'intérêt principal de ce corpus réside justement dans le fait qu'il enregistre les transferts du français observables dans le discours des participants hispanophones et permet d'identifier ceux qu'on détecte à un niveau supra-individuel qui peuvent configurer une variété de contact espagnol-français. Par ailleurs, ce corpus permet l'observation dans la pratique des phénomènes d'accommodation entre les hispanophones de diverses aires dialectales qui cohabitent en Suisse. Les deux types de convergences, le transfert et l'accommodation, pourront être étudiés en lien avec des variables externes (génération, âge d'arrivée en Suisse, autres expériences migratoires, bilinguisme avant la migration, couple francophone ou hispanophone, niveau d'études, représentations et attitudes envers l'espagnol, le français et les autres dialectes espagnols, etc.) recueillies également dans le corpus.

12 Pour ce qui est de la méthodologie, le COLESfran se développe avec la participation de linguistes et d'étudiantees de la Section d'espagnol de l'UNIL, qui réalisent des 
enregistrements vidéo et audio d'entretiens semi-dirigés dans lesquels les participante's s'expriment sur leurs pratiques linguistiques et sociales. Nous disposons de plusieurs questionnaires en fonction de la génération à laquelle appartiennent les personnes interrogées ${ }^{12}$. Pour les participants de la 1ère génération (G1), les questions portent sur leur expérience de la migration et sur l'histoire de leur vie et de leur intégration linguistique en Suisse. Le questionnaire est structuré en 13 modules thématiques:
1. l'étape précédant l'émigration et la décision d'émigrer
2. l'arrivée en Suisse
3. la famille
4. les langues : compétences, pratiques et représentations
5. les amis hispanophones et francophones
6. les relations avec les autres hispanophones
7. les loisirs
8. la consommation des médias
9. la vie associative
10. les intérêts politiques
11. la nationalité
12. les voyages et les contacts avec le pays d'origine
13. les éventuels projets de retour

13 Dans le cas des générations suivantes (G2 et G3), le questionnaire est adapté avec des questions concernant de façon spécifique ces membres de la communauté hispanophone, telles que la manière dont ils ont acquis l'espagnol (avec ou sans soutien de cours de langue et de culture d'origine), leur usage des langues avec la famille, leurs habitudes de lecture et d'écriture dans la langue d'origine, leurs attitudes envers les deux pays et les deux langues, leurs relations avec les autres hispanophones en Suisse, avec les personnes et les institutions dans le pays d'origine, etc. Tant dans le cas de la G1 que des générations suivantes, nous nous intéressons à la connaissance et à la pratique d'autres langues d'Espagne et d'Amérique par les participants, afin d'intégrer dans la recherche cette variable, que nous considérons très pertinente pour l'interprétation du reste des données sociolinguistiques.

Compte tenu des normes récentes dans le domaine scientifique concernant le traitement des données personnelles, nous avons formalisé par écrit, depuis 2019, un document avec des informations sur les objectifs et la nature du projet ainsi qu'un formulaire d'autorisation des participants à enregistrer les entretiens et à utiliser les données de manière anonyme par la suite dans le cadre de notre recherche.

À ce jour, nous avons réalisé une centaine d'entretiens avec des Espagnols et des Hispano-américains de la première et de la deuxième génération dans les proportions suivantes : 22 pour la G1 espagnole et 50 pour la G1 américaine (principalement des Colombiens, des Chiliens et des Mexicains, mais aussi des participants d'Argentine, de Bolivie, d'Équateur, du Pérou, du Panama, de la République dominicaine, de Cuba et du Venezuela) ; 18 pour la $\mathrm{G} 2$ espagnole et 10 pour la G2 américaine.

16 En plus de ces entretiens, le COLESfran en inclut d'autres à des fins spécifiques : par exemple, en 2015, une étudiante a mené deux entretiens avec des personnes parlant le judéo-espagnol nées à Istanbul et à Vienne ; en 2019, un étudiant a interrogé des élèves 
et des enseignants des Aulas de Lengua y Cultura Españolas (ALCE) à Lausanne pour explorer certains aspects de cette formation, et nous avons régulièrement interrogé des responsables d'associations et de centres espagnols (le centre d'espagnol de Bussigny, le centre asturien de Renens, ou les anciens centres García Lorca, Promoción da cultura galega et Grupo de Teatro Español, tous à Lausanne). L'équipe du COLESfran a également fait quelques incursions en Suisse italienne, dans le canton du Tessin, où en 2014 nous avons réalisé quatre interviews exploratoires avec une Espagnole, deux Argentins et une Mexicaine.

17 Les interviews du COLESfran ne suivent pas un format unique. Parfois, c'est un groupe qui réalise l'interview et parfois un seul enquêteur. A leur tour, les personnes interrogées peuvent être une personne seule ou un couple (mari et femme, amis, membres de G1 et G2), selon les circonstances naturelles du moment. La durée de nombreux entretiens est d'environ une heure, mais elle peut dépasser les deux heures, en fonction de la disponibilité et de l'intérêt des informateurs à détailler leurs réponses. Jusqu'à présent, la plupart des entretiens ont eu lieu en présentiel dans le domicile de l'informateur ou dans les salles de cours de l'UNIL, mais en 2020, dans le cadre du semi-confinement produit par le coronavirus, nous avons commencé à mener des entretiens par vidéoconférence, impliquant seulement un interviewer et une personne interviewée. Les interviews sont enregistrées sur vidéo et/ou audio et ensuite transcrites. La tâche de transcription est très coûteuse en temps et nécessite une révision par deux membres de l'équipe pour assurer la meilleure qualité possible du corpus. Actuellement, environ un quart du corpus est transcrit dans sa première version, en attente d'une double révision.

Afin d'optimiser ce travail de transcription, nous avons l'intention d'utiliser dans la prochaine étape une technique qui donne d'excellents résultats au COLEM (Corpus oral de la lengua española en Montreal): le off-line respeaking associé à un programme de reconnaissance automatique de la parole, méthode utilisée pour le sous-titrage en direct à la télévision, qui en théorie réduit à trois heures la transcription d'une heure d'interview ${ }^{13}$. Une telle performance serait sans doute inférieure dans le cas du COLESfran - car dans notre corpus l'intervention de plusieurs intervieweurs et interviewés provoque fréquemment des chevauchements de parole assez laborieux à transcrire -, mais le gain de temps prévisible par rapport à la durée actuelle d'une transcription d'une heure (environ 30 heures de travail) contribuerait sensiblement au succès du projet et mérite bien l'investissement en formation et en programmes informatiques.

19 Pour les étapes suivantes d'alignement du texte avec le son, d'annotation morphosyntaxique, de lemmatisation du texte et de sélection d'un moteur de recherche pour récupérer les informations de la base de données, nous réfléchissons actuellement à la technologie à choisir au sein de la nouvelle fédération LAMIE (Fédération internationale pour l'étude sociolinguistique des LAngues des MIgrants Espagnols en France et dans l'Europe francophone), composée de membres des universités de Paris 8 , Perpignan, Paris 3, Lyon 2, Louvain, Antonio de Nebrija et de l'Université Autonome de Barcelone. Cette équipe prévoit actuellement de réaliser des interviews et de compiler des corpus de caractéristiques similaires à celles du COLEM et du COLESfran afin de créer ensemble une base de données interopérable pour l'étude de l'effet sur l'espagnol du contact avec le français dans tous les pays francophones où il existe une importante communauté hispanophone. 


\section{Le COLESfran comme outil pédagogique, ses résultats et perspectives}

20 Le corpus est créé en collaboration avec les étudiant·es (principalement du niveau master, mais occasionnellement aussi du bachelor) de la Section d'espagnol de l'UNIL, où depuis 2013 l'auteure de cet article et Elena Diez del Corral Areta proposent régulièrement des cours portant sur la linguistique de la migration et le contact linguistique ${ }^{14}$. Dans ce cadre, les étudiants mènent des entretiens comme travail de terrain avant d'en analyser les contenus à la lumière des théories de la linguistique de la migration, du bilinguisme et du contact. Toutes ces activités sont à la fois pédagogiquement très instructives et stimulantes pour eux. Un des formats déjà classiques des travaux des séminaires de master consiste à analyser les stratégies d'intégration sociolinguistique que manifestent les participants du corpus, en s'appuyant sur les modèles proposés par des auteurs tels que Gugenberger et Moreno Fernández ${ }^{15}$, et à examiner les particularités structurelles de leur discours qui découlent du contact avec le français. D'autres travaux de séminaire s'intéressent à des aspects spécifiques de la sociologie des langues en contact, tels que l'accommodation dialectale entre locuteurs de différentes variétés, les attitudes linguistiques passées et présentes des locuteurs d'héritage de la Section d'espagnol de l'UNIL et leurs besoins pédagogiques particuliers, ou la transmission intergénérationnelle du galicien dans les familles vivant en Suisse romande.

21 Ces travaux ont trouvé une continuité dans plusieurs mémoires de master: Roxane Morel (Estudio sociolingüístico de la comunidad inmigrante chilena en Lausana, 2015) a étudié les attitudes linguistiques et la variété de contact de huit membres de la plus ancienne communauté hispanophone de ce pays, en raison de leur exil entre 1973 et 1988, sous la dictature de Pinochet; Mathieu Ballif (Estudio sociolingüistico de españoles de segunda generación en el cantón de Vaud y del Valais, 2015) a exploré auprès de six membres de la deuxième génération d'Espagnols des questions sociales d'identité, d'utilisation de la langue et d'attitudes envers les langues, et s'est aussi intéressé aux phénomènes de transfert et de changement de code; Johanna Henao (Estudio sociolingüístico de emigrantes colombianos en Nueva York y Suiza francófona, 2016) a analysé et comparé les compétences et les attitudes dans la langue d'origine et dans la langue d'accueil des Colombiens de première et de deuxième génération qui ont émigré à New York et en Suisse romande (six participants dans chaque catégorie, vingt-quatre au total), en considérant les différences dans les politiques d'intégration et la valeur du multilinguisme dans ces pays; Mariola Broccard (Hacia una descripción morfosintáctica y léxica de la variedad de contacto español francés en Suiza, 2018) a interviewé sept hispanophones de première génération, d'origines différentes (Colombie, République dominicaine, Argentine, Chili, Panama, Espagne et Mexique), qui résident en Suisse romande depuis plusieurs décennies, afin d'examiner les caractéristiques communes de leur lexique et de leur grammaire qui résulteraient d'un contact intense avec le français, dans le but d'identifier les traits propres à la variété de l'espagnol en contact avec le français; enfin, Leslie Bezençon (El español de residentes mexicanos en la Suiza francófona: fenómenos de contacto en dos generaciones, 2019) a examiné le discours de douze migrants mexicains, huit de la première génération et quatre de la génération "1,5" (ils avaient entre six et huit ans lorsqu'ils ont migré en Suisse), afin d'identifier les 
transferts et le code-switching dans les deux groupes, ainsi que la conscience linguistique de ces locuteurs.

Les interviews du COLESfran servent également de base de données de recherche pour les linguistes de la Section d'espagnol de l'UNIL. Elena Diez del Corral Areta $(2018)^{16}$, à partir de dix entretiens avec des migrants colombiens vivant dans les cantons de Genève, Neuchâtel et Vaud, a centré son analyse sur leurs déclarations identitaires afin de découvrir s'ils se définissaient culturellement et linguistiquement de manière hybride, comme bilingues et biculturels. Mónica CASTILLO LLuCH (2019) ${ }^{17}$, afin de décrire le paysage linguistique en espagnol de la ville de Lausanne avec une profondeur historique, c'est-à-dire en documentant la raison et le moment où chaque signe est apparu dans cette langue, a également utilisé une série d'entretiens avec des entrepreneurs et des responsables de centres culturels, grâce auxquels elle a pu situer les signes dans cette langue au moment de leur production et accéder à l'histoire de la communauté immigrée hispanophone de la ville.

Les mémoires de master des étudiants et nos articles de recherche peuvent être consultés en ligne sur le site www.colesfran.ch, que nous avons créé pour héberger divers contenus: une bibliographie sur l'espagnol en Suisse avec certaines références directement téléchargeables (dont les mémoires de master des étudiants et nos articles mentionnés), des informations sur la communauté hispanophone de Suisse (démographie, cours de langue et culture d'origine, paysage linguistique), des annonces des différentes manifestations scientifiques que nous organisons à l'UNIL en relation avec l'espagnol en Suisse, et le menu que nous souhaitons développer dans les prochaines années, qui est celui consacré au COLESfran. Actuellement, ce menu comprend des informations sur les objectifs, la méthodologie, les campagnes et quelques fragments du corpus, et nous travaillons pour y rendre accessible notre base de données COLESfran d'ici 2025.

Les perspectives de travail de notre équipe incluent la collaboration avec d'autres projets pour l'étude du contact entre l'espagnol et le français, parmi lesquels le Corpus oral de la lengua española en Montreal (COLEM), dirigé par le professeur Enrique Pato, occupe la place la plus importante ${ }^{18}$. Un exemple de collaboration avec le COLEM est le projet d'étude des attitudes linguistiques, des identités et des dynamiques de conservation ou perte de l'espagnol auprès des communautés hispanophones des villes de Lausanne, Montréal et New York, que Vanessa Casanova prépare pour sa période post-doctorale à partir de l'automne 2021. Par ailleurs, l'équipe du COLESfran de l'Université de Lausanne est actuellement associée à la création de la fédération LAMIE déjà évoquée, qui vise à étudier les langues de l'immigration espagnole et de ses descendants en France et dans les autres pays européens francophones. Au cours des cinq prochaines années, nous nous fixons comme objectifs du COLESfran d'équilibrer la base de données actuelle en la complétant par des entretiens pour les nationalités les moins représentées dans le corpus et, en même temps, de réaliser toutes les opérations qui permettront de consulter le corpus en ligne : sa transcription, l'alignement du texte et du son, l'étiquetage et son intégration dans un moteur de recherche.

Enfin, nous estimons que, même si le COLESfran a été conçu comme un corpus de données pour les études sociolinguistiques, il peut être utilisé par d'autres disciplines des sciences sociales et humaines qui s'intéressent à la communauté espagnole et hispano-américaine vivant en Suisse entre 1960 et aujourd'hui, et qui utilisent aussi régulièrement les récits de vie comme base empirique pour leurs recherches. D'autre 
part, nous sommes également très intéressés par le matériel recueilli par d'autres spécialistes (notamment des historiens et des sociologues) qui étudient ces communautés en Suisse. Pour ne citer que deux exemples, le corpus des émissions du programme Tele-revista de la Télévision Suisse entre 1973 et $1989^{19}$, grâce auquel Anthony Roduit a pu réaliser un mémoire de master pour examiner l'ensemble des contenus traitant des questions linguistiques (Estudio de las actitudes y representaciones lingüísticas entre la comunidad hispanohablante en Suiza a partir del corpus del programa de la RTS Tele-revista, 2017) et le volume Secondos-Secondas ${ }^{20}$, qui rassemble les résultats d'une vaste étude sur les enfants d'immigrants italiens et espagnols pour analyser leurs dynamiques d'intégration et de mobilité sociales et leurs stratégies identitaires, qui nous sert de source pour contextualiser toutes nos études sur la deuxième génération d'hispanophones.

\section{Conclusions}

Dans cet article ont été exposés les objectifs, méthodologie, état actuel, résultats, perspectives et défis du Corpus oral de la lengua española en la Suiza francófona (COLESfran), qui réunit des témoignages oraux de la communauté hispanophone en Suisse romande, recueillis au moyen d'interviews semi-dirigés portant sur l'expérience migratoire et les pratiques sociales et linguistiques des participants.

La communauté hispanophone a un poids numérique considérable en Suisse depuis les années 1960, et plus spécifiquement en Suisse romande, où notamment le nombre d'Espagnols est très élevé par rapport à la population totale, surtout dans les villes de Genève et de Lausanne. Le COLESfran est une base de données pour l'étude des pratiques linguistiques de la communauté hispanophone résidant dans cette zone linguistique de la Confédération helvétique avec une double perspective : du point de vue de la sociologie des langues dans le contexte de la migration et du point de vue sociolinguistique, en analysant les phénomènes de variation de l'espagnol de ces locuteurs dus au contact avec le français. Une centaine d'entretiens avec des Espagnols et des Hispano-américains de première et deuxième générations intègrent à ce jour le corpus, qui se développe en collaboration entre linguistes spécialistes et étudiant·es au sein d'enseignements de la Section d'espagnol de l'UNIL consacrés à la linguistique de la migration et du contact. Dans les pages qui précèdent, une liste a été dressée des divers travaux des spécialistes et des étudiant·e's déjà menés sur la base des contenus des interviews existants.

Ont été également présentés les divers enjeux actuels du projet, dont tout d'abord celui d'améliorer la performance du travail de transcription grâce à l'emploi des nouvelles technologies. Nous envisageons enfin de réaliser l'alignement du texte de ces transcriptions avec le son, l'annotation morphosyntaxique, la lemmatisation du texte et la sélection d'un moteur de recherche pour récupérer les informations de la base de données, de commun accord avec les partenaires de la nouvelle fédération LAMIE, afin de créer un corpus commun pour l'étude de l'espagnol en contact avec le français dans tous les pays francophones où il existe une importante communauté hispanophone. 


\section{BIBLIOGRAPHIE}

Claudio BolzMAN, Rosita FIBBI, Marie VIAL, Secondas-Secondos. Le processus d'intégration des jeunes adultes issus de la migration espagnole et italienne en Suisse, Zurich, Seismo, 2003.

Luís Manuel CALVO SALGADO, « La emigración española en Suiza desde los años sesenta y la primera etapa de las relaciones bilaterales en materia de política migratoria ", Joseba de la Torre et Gloria Sanz Lafuente (éds.), Migraciones y coyuntura económica del franquismo a la democracia, Zaragoza, Prensas Universitarias de Zaragoza, 2008, p. 289-316.

Luís Manuel CALVO SALGADO et Moisés PRIETO LóPEZ, « Microhistoria del comienzo de la emigración española en Suiza : lucha política y una película prohibida », Veronica Orazi, Federica Cappelli, Iole Scamuzzi et Barbara Greco (éds.), Trayectorias literarias hispánicas : tradición, innovación y nuevos paradigmas, Roma, AISPI Edizioni, 2019, p. 329-343.

Luís Manuel CALVO SALGADO, Concha LANGA NUÑo, Moisés PRIETO LóPEZ, Tele-revista y la Transición : un programa de la televisión suiza para emigrantes españoles (1973-1989), Madrid/ Frankfurt, Iberoamericana/ Vervuert, 2015.

Mónica CASTILLO LLUCH, « Reading diachrony in linguistic landscape synchrony: Spanish in Lausanne », Mónica Castillo Lluch, Rolf Kailuweit et Claus D. Pusch, (éds.), Linguistic Landscape Studies: The French Connection, Freiburg im Breisgau, Rombach (Freiburger Romanistische Arbeiten vol. 15), 2019, p. 59-93.

Mónica CASTILLO LLUCH, « El Corpus oral de la lengua española en la Suiza francófona (COLESfran) », Boletín hispánico helvético, nº 35-36 (primavera-otoño 2020), p. 289-316.

Mónica CASTILLO LLUCH, El español en la Suiza francófona. COLESfran (Corpus oral de la lengua española en la Suiza francófona), <URL : http://wp.unil.ch/colesfran/cursos-de-lengua-de-origen/>.

Mónica CASTILLO LLUCH, « Reading diachrony in linguistic landscape synchrony: Spanish in Lausanne », Mónica Castillo Lluch, Rolf Kailuweit et Claus D. Pusch, (éds.), Linguistic Landscape Studies: The French Connection, Freiburg im Breisgau, Rombach (Freiburger Romanistische Arbeiten vol. 15), 2019, p. 59-93.

Elena DIEZ DEL CORRAL ARETA, « Me siento muy poquititico suiza: identificación bilingüe y bicultural en inmigrantes colombianos de la Suiza francófona », Lengua y migración, 10-2 (2018), p. 33-55. GOBIERNO DE ESPAÑA, MINISTERIO DE EDUCACIÓN Y FORMACIÓN PROFESIONAL, El mundo estudia español 2018, 2018 [consulté le 29.11.2020] <URL : https://sede.educacion.gob.es/publiventa/el-mundo-estudiaespanol-2018/ensenanza-lengua-espanola/22602>.

Eva GUGENBERGER, « Aculturación e hibrididad lingüística: Propuesta de un modelo teóricoanalítico para la lingüística de la inmigración », Revista Internacional de Lingüística Iberoamericana : Lengua y migración en el mundo hispanohablante, $\mathrm{n}^{\circ} \mathrm{V}, 2$ (2007), p. 22-45.

INSTITUTO NACIONAL DE ESTADÍSTICA (INE), « Estadística del padrón de españoles residente en el extranjero. Datos a 1-1-2020 » [consulté le 28.11.2020] <URL : https://www.ine.es/jaxi/ Datos.htm ?path $=/$ t20/p85001/a2020/10/\&file =01001.px\# !tabs-tabla>.

FrancisCO MORENO FERNÁNDEZ, « Integración sociolingüística en contextos de inmigración: marco epistemológico para su estudio en España », Lengua y Migración, nº 1, 1 (2009), p. 121-156.

OFFICE FÉDÉRAL DE LA STATISTIQUE. OFS, « Bilan de la population résidante permanente étrangère selon la nationalité, en 2019 », publiée par l'OFS en ligne [consultée le 29.11.2020] <URL : https:// 
www.bfs.admin.ch/bfs/fr/home.html> (Trouver des statistiques $>$ Population > Migration et intégration > Population de nationalité étrangère).

Enrique PATO, «El español en contacto con el francés en Quebec y su estudio gracias al Corpus oral de la lengua española en Montreal (COLEM) », Boletín hispánico helvético, nº 35-36 (2020), p. 263-287.

Marimar RUFINO MORALES, «Estudio comparativo de métodos de transcripción para corpus orales: el caso del español », Revista Nebrija de Lingüística Aplicada a la Enseñanza de Lenguas, nํ 14.29 (2020).

Verónica SÁNCHEZ ABCHI, « Spanish as a heritage language in Switzerland », Kim Potowski (éd.), The Routledge Handbook of Spanish as a Heritage Language, London/ New York, Routledge, 2018, p. 504-516.

\section{NOTES}

1. Cf. Mónica CAStillo LluCh, «El Corpus oral de la lengua española en la Suiza francófona (COLESfran) », Boletín hispánico helvético, no 35-36 (primavera-otoño 2020), p. 289-316, article dans lequel est présenté pour la première fois ce corpus.

2. Selon les données de l'Instituto nacional de estadística (INE) («Estadística del padrón de españoles residente en el extranjero. Datos a 1-1-2020»:

<URL : https://www.ine.es/jaxi/Datos.htm ?path =/t20/p85001/a2020/10/\&file =01001.px\# !tabstabla> [consulté le 28.11.2020]. Les pays étrangers dans lesquels le nombre de résidents espagnols dépasse cent mille personnes sont au nombre de dix selon l'INE : outre les trois pays européens précités, l'Argentine (473.519), le Brésil (133.188), Cuba (147.617), les États-Unis d'Amérique (167.426), le Mexique (144.553) et le Vénézuela (142.302). Les données statistiques sur lesquelles s'appuie notre étude, sauf indication contraire, sont issues de l'office fédéral de la Statistique suisse (OFS). Pour l'ensemble des données démo-linguistiques suivantes, $c f$. les sources dans Mónica CASTILlO LLUCH, «Reading diachrony in linguistic landscape synchrony: Spanish in Lausanne », dans Mónica Castillo Lluch, Rolf Kailuweit et Claus D. Pusch, (éds.), Linguistic Landscape Studies: The French Connection, Freiburg im Breisgau, Rombach (Freiburger Romanistische Arbeiten vol. 15), 2019, p. 59-93 (p. 64-65). Les données pour 2019 sont issues de la feuille Excel "Bilan de la population résidante permanente étrangère selon la nationalité, en 2019 ", publiée par l'OFS en ligne <URL : https://www.bfs.admin.ch/bfs/fr/home.html> (Trouver des statistiques $>$ Population > Migration et intégration > Population de nationalité étrangère) [consultée le 29.11.2020].

3. Selon des données de 2019 de l'OFS, qui ne tiennent compte que des mono-nationaux <URL : https://www.pxweb.bfs.admin.ch/pxweb/fr/px-x-0102010000_104/-/px-x-0102010000_104.px/ ? rxid =ea8203c4-abbf-46a3-a488-db177e99905d> [consulté le 29.11.2020].

4. Je tiens à remercier Amélie de Flaugergues, cheffe de projets à l'OFS, de m'avoir fourni ces informations et de m'avoir guidée dans la recherche d'autres données statistiques.

5. En ligne : <URL : https://www.admin.ch/opc/fr/classified-compilation/19610027/index.html> [consulté le 29.11.2020]. Pour les données qui suivent, cf. Luís Manuel CALvo SALGADo, «La emigración española en Suiza desde los años sesenta y la primera etapa de las relaciones bilaterales en materia de política migratoria », Joseba de la Torre et Gloria Sanz Lafuente (éds.), Migraciones y coyuntura económica del franquismo a la democracia, Zaragoza, Prensas Universitarias de Zaragoza, 2008, p. 289-316 et Luís Manuel CALVo SALGADO et Moisés PRIETO LóPEZ, « Microhistoria del comienzo de la emigración española en Suiza : lucha política y una película prohibida", 
Veronica Orazi, Federica Cappelli, Iole Scamuzzi et Barbara Greco (éds.) : Trayectorias literarias hispánicas : tradición, innovación y nuevos paradigmas, Roma, AISPI Edizioni, 2019, p. 329-343.

6. Les Hispano-américains étaient au nombre de 7.178 à la fin de 1980 et 11.840 à la fin de 1990 , mais déjà 21.369 à la fin de 2000.

7. Par ailleurs, dans les statistiques suisses ne figurent pas parmi les Hispano-américains ceux qui sont arrivés en Suisse avec un passeport helvétique, à la suite de la migration de leurs ancêtres (c'est le cas de nombre d'Argentins, par exemple).

8. Pour plus de détails sur les nationalités des Hispano-américains vivant en Suisse, $c f$. le graphique «Evolución de la población hispanoamericana en Suiza » sur le site <URL : https:// www.mapaespanolsuiza.org/grafico-9>. [consulté le 28.11.2020]. Sur ce même site <URL : https:// www.mapaespanolsuiza.org/>, on trouve des graphiques supplémentaires sur d'autres aspects de l'espagnol en Suisse.

9. En ligne : <URL : https://www.bfs.admin.ch/bfs/fr/home/statistiques/themes-transversaux/ analyses-spatiales/niveaux-geographiques/regions-analyse.assetdetail.2543293.html> [consulté le 29.11.2020].

10. Cf. l'étude de Verónica SÁNCHEZ ABCHI, "Spanish as a heritage language in Switzerland », Kim Potowski (éd.), The Routledge Handbook of Spanish as a Heritage Language, London/ New York, Routledge, 2018, p. 504-516, ainsi que notre site web El español en la Suiza francófona. COLESfran (Corpus oral de la lengua española en la Suiza francófona), <URL : http://wp.unil.ch/colesfran/cursosde-lengua-de-origen/> [consulté le 29.11.2020]. L'importance accordée par les familles espagnoles au maintien de leur langue d'origine en Suisse se traduit par le chiffre de 4.400 élèves inscrits dans les Aulas de lengua y cultura española en 2018 dans ce pays. Il s'agit du chiffre le plus élevé de tous les pays dans lesquels ces cours sont offerts par le gouvernement espagnol (suivi par celui de 2.878 élèves en Allemagne, 2.400 au Royaume Uni et 2.088 en France). Cf. Gobierno de España, Ministerio de Educación y formación profesional, El mundo estudia español 2018, 2018 [consulté le 29.11.2020] <URL: https://sede.educacion.gob.es/publiventa/el-mundo-estudia-espanol-2018/ ensenanza-lengua-espanola/22602>.

11. Dans Mónica CASTILlo LLUCH, «El Corpus oral de la lengua española en la Suiza francófona (COLESfran) ", Boletín hispánico helvético, ํo 35-36 (primavera-otoño 2020), p. 289-316 (p. 302-307), est dressé un inventaire des phénomènes de contact espagnol-français documentés dans le COLESfran.

12. Sur notre site web El español en la Suiza francófona. COLESfran (Corpus oral de la lengua española en la Suiza francófona), <URL : https://wp.unil.ch/colesfran/cuestionarios/> [consulté le 29.11.2020] on peut découvrir deux de nos questionnaires (G1 et G2).

13. Cf. Marimar RUFINO MORALES, «Estudio comparativo de métodos de transcripción para corpus orales : el caso del español », Revista Nebrija de Lingüística Aplicada a la Enseñanza de Lenguas, 14.29 (2020).

14. La liste des étudiant·e's ayant collaboré au COLESfran se trouve sur notre site web El español en la Suiza francófona. COLESfran (Corpus oral de la lengua española en la Suiza francófona), <URL : https:// wp.unil.ch/colesfran/colaboradores/> [consulté le 29.11.2020].

15. Eva GUGENBERGER, «Aculturación e hibrididad lingüística: Propuesta de un modelo teóricoanalítico para la lingüística de la inmigración », Revista Internacional de Lingüística Iberoamericana : Lengua y migración en el mundo hispanohablante, V : 2 (2007), p. 22-45 ; Francisco MORENO FERNÁNDEZ, «Integración sociolingüística en contextos de inmigración: marco epistemológico para su estudio en España », Lengua y Migración, 1 : 1 (2009), p. 121-156.

16. Elena DIEZ DEL CORRAL ARETA, «Me siento muy poquititico suiza: identificación bilingüe y bicultural en inmigrantes colombianos de la Suiza francófona ", Lengua y migración, 10-2 (2018), p. 33-55.

17. Mónica CASTILLO LLUCH, «Reading diachrony in linguistic landscape synchrony: Spanish in Lausanne », Mónica Castillo Lluch, Rolf Kailuweit et Claus D. Pusch, (éds.), Linguistic Landscape 
Studies: The French Connection, Freiburg im Breisgau, Rombach (Freiburger Romanistische Arbeiten vol. 15), 2019, p. 59-93.

18. Cf. Enrique PATO, «El español en contacto con el francés en Quebec y su estudio gracias al Corpus oral de la lengua española en Montreal (COLEM) », Boletín hispánico helvético, 35-36 (2020), p. 263-287.

19. Luís Manuel CAlVo SALGADO, Concha LANGA NUÑo, Moisés PRIETO LóPEZ, Tele-revista y la Transición : un programa de la televisión suiza para emigrantes españoles (1973-1989), Madrid/ Frankfurt, Iberoamericana/ Vervuert, 2015.

20. Claudio BolzMAn, Rosita FIBBI, Marie VIAL, Secondas-Secondos. Le processus d'intégration des jeunes adultes issus de la migration espagnole et italienne en Suisse, Seismo, Zurich, 2003.

\section{RÉSUMÉS}

Cet article présente le Corpus oral de la lengua española en la Suiza francófona (COLESfran), qui depuis 2013 recueille des témoignages d'hispanophones résidant en Suisse romande dans le but d'analyser aussi bien le processus d'intégration linguistique de cette communauté dans ce pays d'accueil que l'influence que le français exerce sur l'espagnol de ces locuteurs. Après un bref profil démographique et historique de la communauté hispanophone en Suisse (romande) sont exposés les objectifs, la méthodologie du corpus, son état actuel et les défis auxquels il est confronté. Sont également présentés l'intérêt du corpus en tant qu'outil pédagogique, certains de ses résultats et les perspectives actuelles du projet.

This article presents the Corpus Oral de la lengua española en la Suiza francófona (COLESfran), which since 2013 has been collecting testimonies from Spanish speakers living in the French-speaking part of Switzerland with the aim of analysing both the process of linguistic integration of this community in the host country and the influence that French exerts on the Spanish of these speakers. After a brief demographic and historical profile of the Spanish-speaking community in (French-speaking) Switzerland, the objectives, the methodology of the corpus, its current status and the challenges it faces are presented. The interest of the corpus as a teaching tool, some of its results and the current prospects of the project are also presented.

\section{INDEX}

Keywords : Spanish-French contact, oral corpus, migration, Spanish in Switzerland

Mots-clés : contact espagnol-français, corpus oral, migration, espagnol en Suisse

\section{AUTEUR}

\section{MÓNICA CASTILLO LLUCH}

Université de Lausanne 